Check for updates

Cite this: RSC Adv., 2019, 9, 3157

\title{
Exploring the temperature effect on hole transport properties in organic bulk heterojunctions
}

\author{
Bixin Li, (D)* Shiyang Zhang and Xianglin Li
}

The temperature effect on hole transport in representative organic bulk heterojunctions has been explored. Two model systems, namely, copper phthalocyanine (CuPc):fullerene $\left(\mathrm{C}_{60}\right)$, and zinc phthalocyanine $(\mathrm{ZnPc}): \mathrm{C}_{60}$, are chosen as case studies. The current-voltage $(I-V)$ characteristics have been measured in hole-only configurations in the temperature range of $100-280 \mathrm{~K}$ and the temperature-dependent hole transport process has been revealed. At high voltages and temperatures from 180 to $280 \mathrm{~K}$, charge transport is governed by the trapping model with space charge limited conduction in the presence of an exponential trap distribution. However, at temperatures below 180 $K$, the mobility model with field-dependent mobility fits the experimental data well, indicating that charge conduction occurs through a hopping mechanism. The extended state and localized state affected by temperature are responsible for hole transport in high and low temperature ranges, respectively.

Received 5th December 2018

Accepted 14th January 2019

DOI: $10.1039 / \mathrm{c} 8 \mathrm{ra10006c}$

rsc.li/rsc-advances

BHJs by the organic thin film transistor (OTFT) technique. ${ }^{11-14}$ However, charge carriers move in the direction parallel to thin films within a very thin insulator-organic interface region, which is different from those movements in OSCs. Notably, space charge limited current (SCLC) measurement, which requires thinner film thickness, is a powerful and convenient method to evaluate the charge transport process in pristine film or BHJs. ${ }^{15-19}$

The charge carrier transport process is always accompanied by the capture of some carriers in localized states, which might act as recombination centres or traps. Generally, there are two models to describe the transport mechanism, i.e.: (i) the trapping model, which assumes a certain distribution of traps in energy spaces and (ii) the mobility model, which assumes an electric field dependence of mobility. ${ }^{20,21}$ The effect of cadmium selenide (CdSe) quantum dots on hole transport in P3HT has been studied over a wide temperature range and the transport mechanism differs. A comparative study on CuPc: $\mathrm{C}_{60}$ and zinc phthalocyanine (ZnPc): $\mathrm{C}_{60}$ BHJs revealed trap-limited conduction above $180 \mathrm{~K} .{ }^{19}$ Lou et al. investigated the charge transport and electronic traps in P3HT:PCBM BHJ based on current-voltage $(I-V)$ measurement in the temperature range 213-293 K.15 Nevertheless, the study of carrier transport in organic BHJs under a wider temperature is still a matter of debate.

In this work, steady temperature-dependent $I-V$ measurements were used to comprehensively investigate the hole transport properties in CuPc: $\mathrm{C}_{60}$ and $\mathrm{ZnPc}: \mathrm{C}_{60} \mathrm{BHJs}$ from 100 to $280 \mathrm{~K}$. By analyzing the $I-V$ curves, we observe that the charge transport mechanism shows a transition from the mobility model at low temperature to the trap model at high temperature

Department of Science Education, Laboratory of College Physics, Hunan First Normal University, Changsha 410205, People's Republic of China. E-mail: lbxin86@hotmail. com 
for both organic BHJs. The origin of the difference caused by temperature is discussed in detail.

\section{Experimental details}

CuPc and ZnPc were purchased from Alfa Aesar and purified by temperature gradient sublimation. The hole-only devices were constructed in the sandwiched configuration of ITO/ PEDOT:PSS $(40 \mathrm{~nm}) / \mathrm{CuPc}: \mathrm{C}_{60}$ or $\mathrm{ZnPc}_{6} \mathrm{C}_{60}(1: 1,120 \mathrm{~nm}) /$ $\mathrm{MoO}_{3}(5 \mathrm{~nm}) / \mathrm{Al}$, where ITO is indium-tin-oxide, PEDOT:PSS is poly(ethylene-dioxythiophene):polystyrenesulfonate (CLEVIOS $\mathrm{P}$ VP Al 4083, H. C. Stark), and $\mathrm{MoO}_{3}$ is molybdenum oxide. PEDOT:PSS and $\mathrm{MoO}_{3}$ were used to facilitate hole injection from ITO and suppress electron injection from $\mathrm{Al}^{22,23}$ A $40 \mathrm{~nm}$ thick layer of PEDOT:PSS was spin coated onto precleaned and plasma-treated ITO coated glass substrates at $3000 \mathrm{rpm}$ and cured at $120{ }^{\circ} \mathrm{C}$ for $30 \mathrm{~min}$ in a vacuum. After drying, the organic $\mathrm{BHJ}$ were deposited under a high vacuum (pressure $<10^{-4} \mathrm{~Pa}$ ) by a coevaporation process. Al $(\sim 100 \mathrm{~nm})$ electrodes were deposited on the organic films through shadow masks. The deposition rates of the organic materials and $\mathrm{Al}$ electrode were controlled at $2 \AA_{\mathrm{s}^{-1}}$ and $10 \AA_{\mathrm{s}}^{-1}$, respectively. The active area of each device was $16 \mathrm{~mm}^{2}$.

The thickness was controlled in situ by a quarter crystal monitor, and calibrated by a Dektak 6M Profiler (Veeco). The $I-V$ characteristics were carried out using a Keithley 2400 sourcemeter integrated with a vacuum cryostat (Optistat DN-V, Oxford Instruments) and an ITC 601 temperature controller in the temperature range $100-280 \mathrm{~K}$. The devices were presented in a sample holder and liquid nitrogen was used as a cooling medium.

\section{Results and discussion}

Fig. 1 presents the $I-V$ characteristics of the hole-only devices for $\mathrm{CuPc}^{\mathrm{C}} \mathrm{C}_{60}$ and $\mathrm{ZnPc}: \mathrm{C}_{60} \mathrm{BHJs}$ at various temperatures in a log-log scale. It is clearly observable that at relatively high bias voltages, the non-linear $I-V$ curves follow the power law $J \propto V^{m}$ with $m>2$ at $280 \mathrm{~K}$ and that $m$ increases with decreasing temperature. This feature may signify an SCLC current with an exponential distribution of traps in energy and space. Using continuous equation and boundary condition as

$$
\begin{array}{r}
J=q \mu p(x) E(x), \\
V=\int E(x) \mathrm{d} x,
\end{array}
$$

the current density-voltage $(J-V)$ characteristics for the trap model are given by $^{20,24}$

$$
J=q^{1-l} \mu N_{\mathrm{v}}\left(\frac{2 l+1}{l+1}\right)^{l+1}\left(\frac{l}{l+1} \frac{\varepsilon \varepsilon_{\mathrm{o}}}{N_{\mathrm{t}}}\right)^{l} \frac{V^{l+1}}{d^{2 l+1}},
$$

where $q$ is the elementary charge, $\mu$ is the hole mobility, $E(x)$ is the electric field, $l$ is the energy parameter given by $E_{\mathrm{t}} / k T\left(E_{\mathrm{t}}\right.$ is the characteristic trap energy, $k$ is the Boltzman constant), $\varepsilon$ is the dielectric constant of the material, $\varepsilon_{\mathrm{o}}$ is the permittivity of free space, $N_{\mathrm{t}}$ is the total density of hole traps, and $d$ is the thickness of the sample.

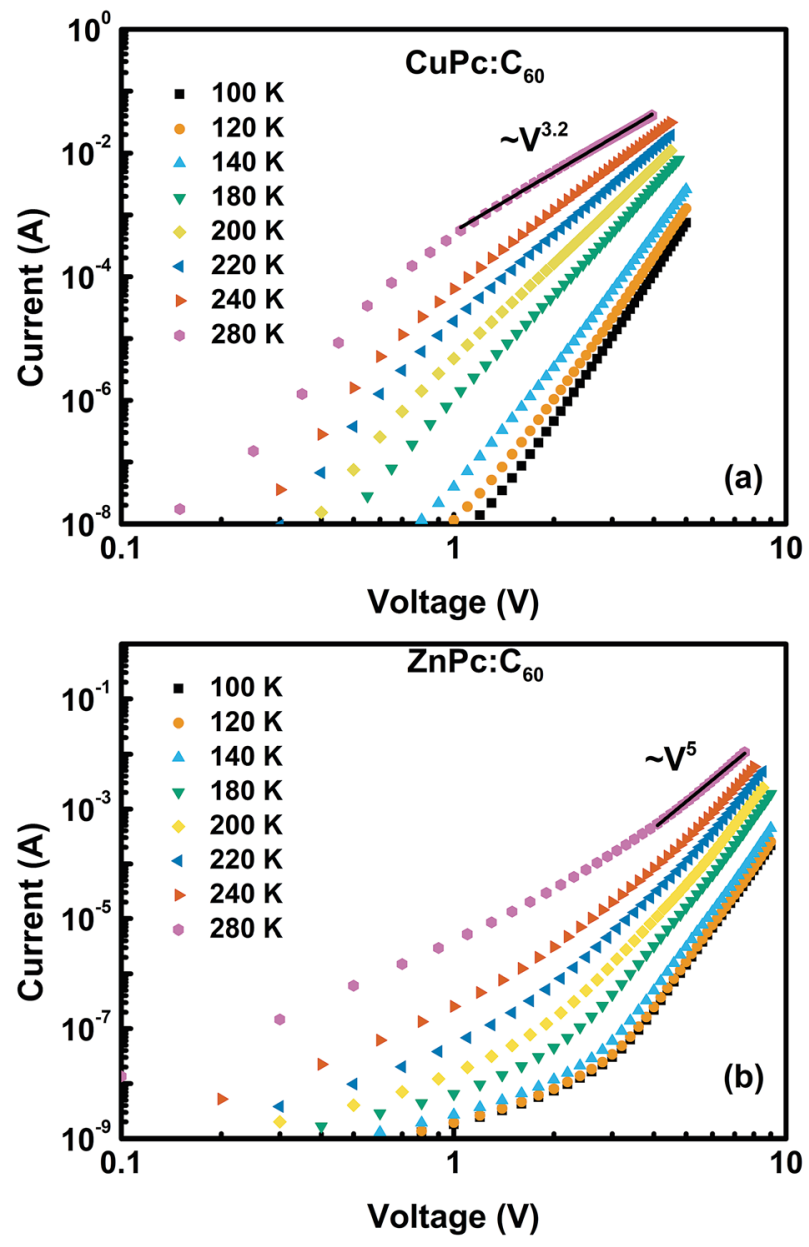

Fig. 1 Current-voltage characteristics of the hole-only devices in the temperature range $100-280 \mathrm{~K}$ based on $\mathrm{BHJ}$ films of (a) $\mathrm{CuPc}_{\mathrm{C}} \mathrm{C}_{60}$ and (b) $\mathrm{ZnPc}: \mathrm{C}_{60}$. Solid lines are linear fits to trap model eqn (3).

In particular, we note that the current density decreases with decreasing temperature. Furthermore, based on the feature of $I$ dependence on $V$ and $T$, two distinct regions have been identified. In the high-temperature region, 180-280 K, the curves tend to converge to a common point. ${ }^{25}$ However, this trend is indistinct under $140 \mathrm{~K}$, the low-temperature region. These results may imply that the transport mechanism differs in the two regions.

Earlier studies have shown that in conducting polymers the charge limited conduction governs the transport either by the trap model at higher temperature or by the mobility model at lower temperature. ${ }^{26}$ In the mobility model, mobility obeys the empirical law ${ }^{21}$

$$
\mu(E, T)=\mu_{\mathrm{o}} \exp \left(-\frac{\Delta_{\mathrm{o}}}{k T}\right) \exp \left[\gamma\left(\frac{1}{k T}-\frac{1}{k T_{\mathrm{o}}}\right) \sqrt{E}\right],
$$

where $\mu_{\mathrm{o}}$ is a constant, $\Delta_{\mathrm{o}}$ is the zero field activation energy, $k$ is the Boltzmann constant, $T$ is the absolute temperature, $\gamma$ is the Poole-Frenkel-like coefficient, and $T_{\mathrm{o}}=600 \mathrm{~K}$.

In order to elucidate the different underlying transport mechanisms, the activation energy was analyzed. Eqn (3) can be simplified to obtain the carrier density and activation energy of traps as ${ }^{25}$ 


$$
J=\frac{q \mu N_{\mathrm{v}} V}{2 d} \exp \left[-\frac{E_{\mathrm{t}}}{k T} \ln \left(\frac{q d^{2} N_{\mathrm{t}}}{2 \varepsilon \varepsilon_{\mathrm{o}} V}\right)\right] .
$$

Eqn (5) provides the Arrhenius dependence of current density on temperature and gives the activation energy as

$$
E_{\mathrm{a}}=\frac{E_{\mathrm{t}}}{k} \ln \left(\frac{q N_{\mathrm{t}} d^{2}}{2 \varepsilon \varepsilon_{\mathrm{o}} V}\right) .
$$

The temperature dependences of the current at an applied voltage for the CuPc: $\mathrm{C}_{60}$ and $\mathrm{ZnPc} \mathrm{C}_{60}$ devices are shown in Fig. 2. The plot shows two distinct linear regions for both films, one at a high-temperature regime and the other at a lowtemperature regime. The slope of the high-temperature regime is definitely larger than that of the low-temperature regime, which signifies that the activation energy is much larger in the high-temperature regime. These results further confirm that the conduction mechanism varies in the two regimes for both films.

Consequently, $I-V$ curves from 100 to $280 \mathrm{~K}$ for both films have been comprehensively analyzed by the trap model using eqn (3) and the mobility model using eqn (4), as shown in Fig. 3 and 4. We can clearly observe that the trap model and mobility model determine the $I-V$ characteristics over different temperature ranges. In Fig. 3(a), the experimental data agrees well with the trap model rather than the mobility model, with the correlation coefficients $r$ being 0.99985 and 0.99881 , respectively. The fitting parameters obtained are $\mu=1.0 \times 10^{-5} \mathrm{~cm}^{2} \mathrm{~V}^{-1} \mathrm{~s}^{-1}, N_{\mathrm{v}}=1.0 \times$ $10^{19} \mathrm{~cm}^{-3}, N_{\mathrm{t}}=2.7 \times 10^{17} \mathrm{~cm}^{-3}$. However, the trap model shows great discrepancy with the experimental data from 100 to $140 \mathrm{~K}$ in Fig. 3(b). Dashed lines represent the theoretically generated curves using the mobility model at different temperatures and they agree well with the experimental data, with the parameters being $\mu_{\mathrm{o}}=4.5 \times 10^{-9} \mathrm{~cm}^{2} \mathrm{~V}^{-1} \mathrm{~s}^{-1}$, and $\gamma=7.0 \times 10^{-5} \mathrm{eV} \mathrm{cm}^{-1 / 2}$ $\mathrm{V}^{-1 / 2}$. In addition, by extrapolating the activation energy at different voltages to zero field, as presented in Fig. 5, the zero field activation energy $\Delta_{\mathrm{o}}$ is found to be $0.096 \mathrm{eV}$.

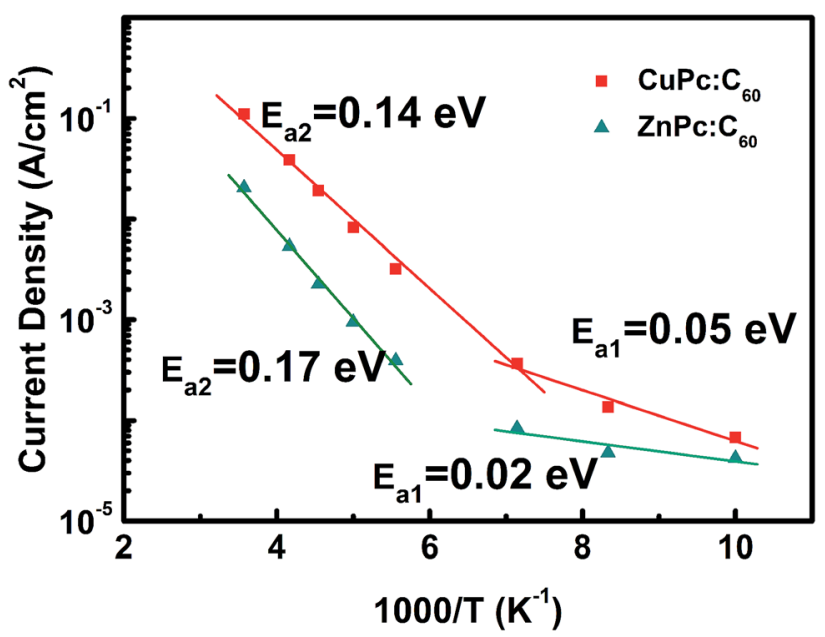

Fig. $2 \mathrm{~J}$ vs. $1000 / T$ plots for CuPc: $\mathrm{C}_{60}$ and $\mathrm{ZnPc}: \mathrm{C}_{60}$ at $3 \mathrm{~V}$ and $6 \mathrm{~V}$, respectively. Solid lines are fits to eqn (5).
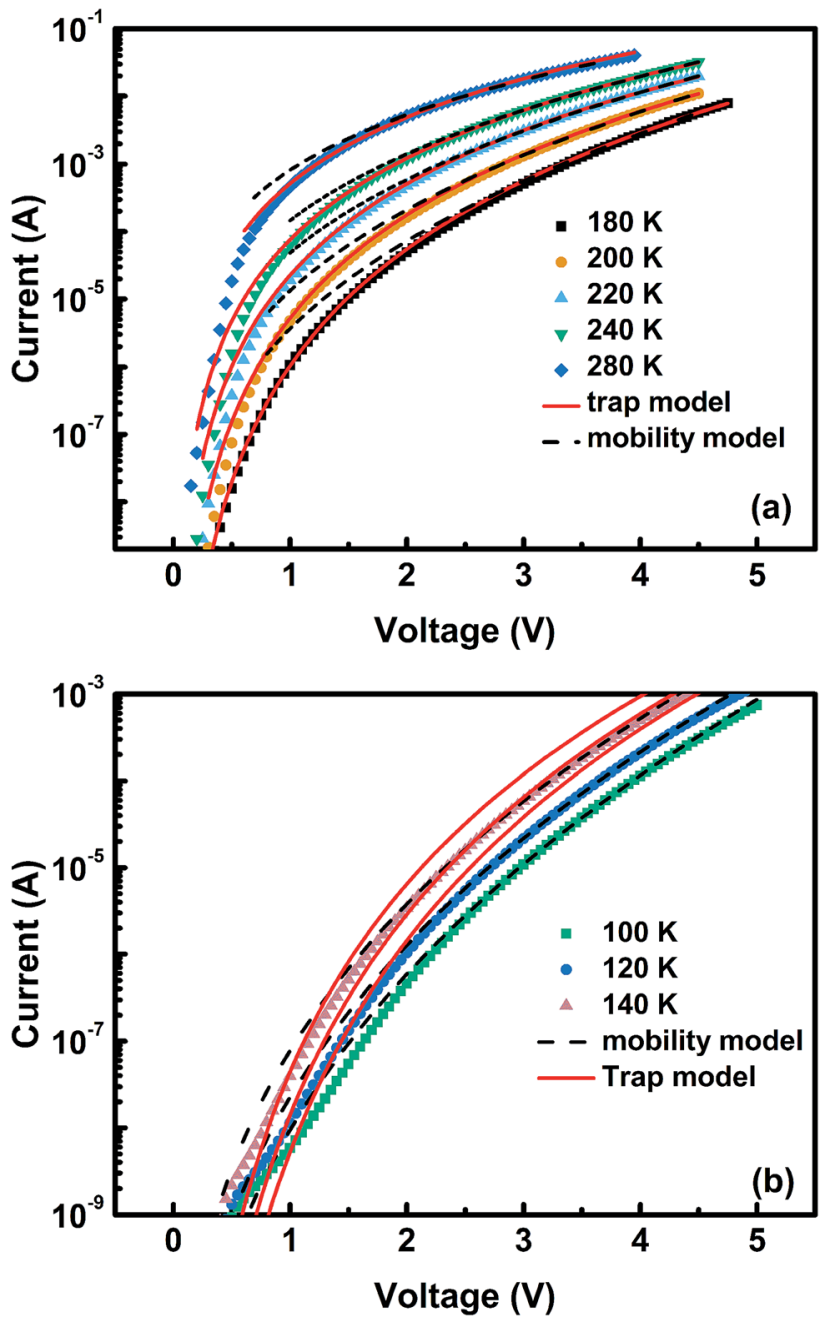

Fig. 3 Current-voltage characteristics of $\mathrm{CuPc}_{\mathrm{P}} \mathrm{C}_{60}$ hole-only devices in (a) the higher temperature range and (b) the lower temperature range. Solid lines and dashed lines are fits to eqn (3) and (4), respectively.

As demonstrated by Fig. 4, a similar phenomenon can also be noted for the $\mathrm{ZnPc}: \mathrm{C}_{60} \mathrm{BHJ}$ film. From 180 to $280 \mathrm{~K}$, the entire set of data fits better with the trap model $(r=0.99882)$ than the mobility model $(r=0.99694)$. The values of the fitting parameters in this case are $\mu=6.2 \times 10^{-6} \mathrm{~cm}^{2} \mathrm{~V}^{-1} \mathrm{~s}^{-1}, N_{\mathrm{v}}=1.0$ $\times 10^{19} \mathrm{~cm}^{-3}$, and $N_{\mathrm{t}}=5.0 \times 10^{17} \mathrm{~cm}^{-3}$. These values are then used to predict the currents at lower temperatures. In contrast, the predicted results clearly overestimate the experimental data over a wide voltage range, while the mobility model shows excellent agreement from $100 \mathrm{~K}$ to $140 \mathrm{~K}$. The parameters obtained are $\mu_{\mathrm{o}}=8.23 \times 10^{-13} \mathrm{~cm}^{2} \mathrm{~V}^{-1} \mathrm{~s}^{-1}, \gamma=5.4 \times 10^{-5} \mathrm{eV}$ $\mathrm{cm}^{-1 / 2} \mathrm{~V}^{-1 / 2}$ and $\Delta_{\mathrm{o}}=0.040 \mathrm{eV}$.

Hence, the temperature is responsible for the different transport mechanisms in BHJs. ${ }^{26}$ It is well known that there are extended states and localized states in the material. Both extended and localized states should play an important role in the transport process in the same way as in inorganic amorphous materials. ${ }^{27}$ In the high-temperature region, a sufficient number of holes are ionized from localized states to extended 

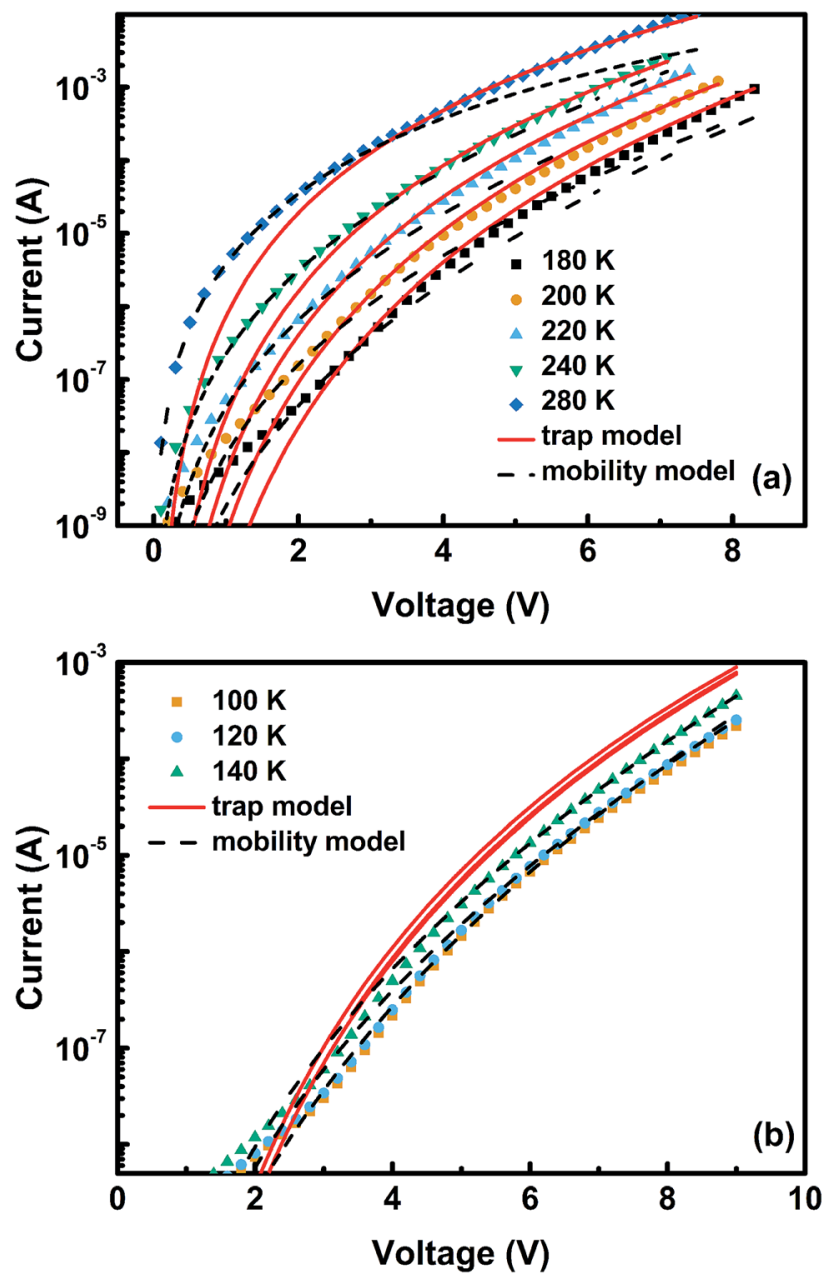

Fig. 4 Current-voltage characteristics of $\mathrm{ZnPc} \mathrm{C}_{60}$ hole-only devices in (a) the higher temperature range and (b) the lower temperature range. Solid lines and dashed lines are fits to eqn (3) and (4), respectively.

states. The current is mainly through the drift of carriers in the extended states with periodic trapping-detrapping in the localized states. ${ }^{28}$ Therefore, the trap model prevails. However,

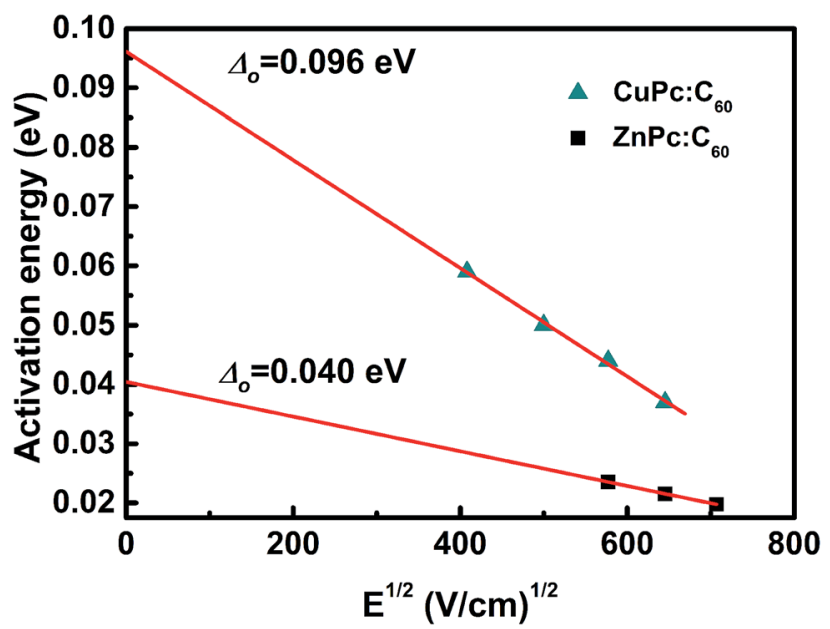

Fig. 5 Low-temperature activation energy versus the square root of the electric field. Solid lines are extrapolations to zero field. at lower temperatures, the carriers in the localized states gain inadequate energy to be excited to the extended states. Thus the conduction takes place through the hopping of carriers from one localized state to another, which shows field and temperature dependence. As a result, the mobility model is justified in the low-temperature region.

\section{Conclusions}

In conclusion, it is demonstrated that temperature plays a significant role in controlling the hole transport process in CuPc: $\mathrm{C}_{60}$ and $\mathrm{ZnPc}: \mathrm{C}_{60} \mathrm{BHJ}$ films. At high temperatures the conduction can be illustrated by the trap model with carriers moving through the extended states. Whereas at low temperatures, the transport is governed by the mobility model with carriers hopping through the localized states. This work assists in gaining a fundamental understanding of the electrical properties of organic $\mathrm{BHJ}$ films that govern device behavior and provides guidance for further device optimization.

\section{Conflicts of interest}

There are no conflicts of interest to declare.

\section{Acknowledgements}

The authors gratefully acknowledge the Hunan First Normal University Research Fund (XYS15N01), the Scientific Research Fund of Hunan Provincial Education Department (17C0331), and the Natural Science Foundation of Hunan Province (Grant No. 2017JJ3046) for their support of this research.

\section{References}

1 D. Meissner and J. Rostalski, Synth. Met., 2001, 121, 15511552.

2 G. Dennler, M. C. Scharber and C. J. Brabec, Adv. Mater., 2009, 21, 1.

3 H. Yin, P. Bi, S. H. Cheung, W. L. Cheng, K. L. Chiu, C. H. Y. Ho, H. W. Li, S. W. Tsang, X. Hao and S. K. So, Sol. RRL, 2018, 2, 1700239.

4 V. Chellappan, G. M. Ng, M. J. Tan, W.-P. Goh and F. Zhu, Appl. Phys. Lett., 2009, 95, 263305.

5 A. Baumann, J. Lorrmann, C. Deibel and V. Dyakonov, Appl. Phys. Lett., 2008, 93, 252104.

6 E. von Hauff, J. Parisi and V. Dyakonov, J. Appl. Phys., 2006, 100, 043702.

7 S. M. Tuladhar, D. Poplavskyy, S. A. Choulis, J. R. Durrant, D. D. C. Bradley and J. Nelson, Adv. Funct. Mater., 2005, 15, 1171-1182.

8 S. A. Choulis, J. Nelson, Y. Kim, D. Poplavskyy, T. Kreouzis, J. R. Durrant and D. D. C. Bradley, Appl. Phys. Lett., 2003, 83, 3812.

9 R. Pacios, J. Nelson, D. D. C. Bradley and C. J. Brabec, Appl. Phys. Lett., 2003, 83, 4764.

10 P. M. Borsenberger and D. S. Weiss, Organic Photoreceptors for Imaging Systems, Dekker, New York, 1993. 
11 A. Opitz, M. Bronner and W. Brütting, J. Appl. Phys., 2007, 101, 063709.

12 M. Bronner, A. Opitz and W. Brütting, Phys. Status Solidi A, 2008, 205, 549-563.

13 A. Opitz, J. Wagner, W. Brütting, A. Hinderhofer and F. Schreiber, Phys. Status Solidi A, 2009, 206, 2683-2694.

14 A. Ko Ko Kyaw, D. Hwan Wang, H.-R. Tseng, J. Zhang, G. C. Bazan and A. J. Heeger, Appl. Phys. Lett., 2013, 102, 163308.

15 Y.-H. Lou, L. Zhang, M.-F. Xu, Z.-K. Wang, S. Naka, H. Okada and L.-S. Liao, Org. Electron., 2014, 15, 299-305.

16 W. Yan-Ping, H. Jin-Ying, C. Jiang-Shan, Q. Xian-Feng, Y. DeZhi, M. Dong-Ge and D. Li-Song, Chin. Phys. Lett., 2016, 33, 027302.

17 A. S. Sarkar and S. K. Pal, J. Phys. D: Appl. Phys., 2015, 48, 445501.

18 M. R. Khan, P. Anjaneyulu, K. S. R. Koteswara Rao and R. Menon, J. Phys. D: Appl. Phys., 2017, 50, 095103.

19 B. Li, J. Chen, D. Yang and D. Ma, Semicond. Sci. Technol., 2011, 26, 115006.
20 K. C. Kao and W. Huang, Electrical Transport in Solids, Pergamon, Oxford, 1981.

21 W. D. Gill, J. Appl. Phys., 1972, 43, 5033-5040.

22 A. Opitz, M. Bronner, J. Wagner, M. Götzenbrugger and W. Brütting, Proc. SPIE, 2008, 7002, 70020J.

23 M. Hoping, C. Schildknecht, H. Gargouri, T. Riedl, M. Tilgner, H. H. Johannes and W. Kowalsky, Appl. Phys. Lett., 2008, 92, 213306.

24 M. A. Lampert and P. Mark, Current Injection in Solids, Academic, New York, 1970.

25 V. Kumar, S. C. Jain, A. K. Kapoor, J. Poortmans and R. Mertens, J. Appl. Phys., 2003, 94, 1283-1285.

26 A. K. Kapoor, S. C. Jain, J. Poortmans, V. Kumar and R. Mertens, J. Appl. Phys., 2002, 92, 3835-3838.

27 P. G. Le Comber and W. E. Spear, Phys. Rev. Lett., 1970, 25, 509-511.

28 C. Li, L. Duan, H. Li and Y. Qiu, J. Phys. Chem. C, 2014, 118, 10651-10660. 